UCRL-ID-124818

\title{
Modeling of MHD Equilibria and Current Profile Evolution during the ERS Mode in TFTR
}

\author{
E.B. Hooper, L.D. Pearlstein, and R.H. Bulmer
}

RECERPED

SEP 09 198S

OSTI

July 18, 1996

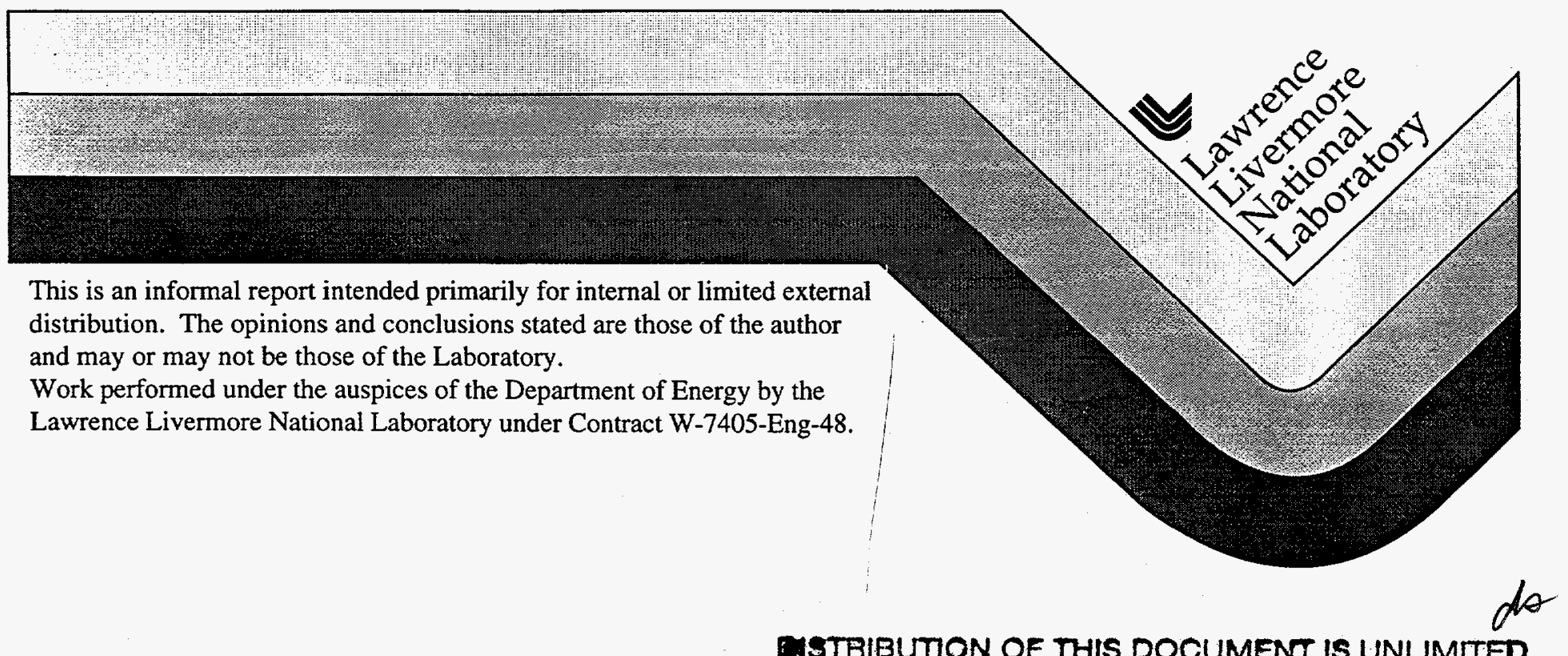

EMSTRIBUTION OF THIS DOCUMENT IS UNLIMITED

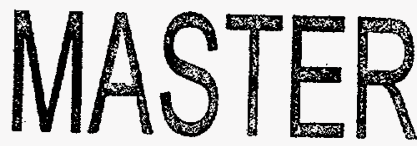




\section{DISCLAIMER}

This document was prepared as an account of work sponsored by an agency of the United States Government. Neither the United States Government nor the University of California nor any of their employees, makes any warranty, express or implied, or assumes any legal liability or responsibility for the accuracy, completeness, or usefulness of any information, apparatus, product, or process disclosed, or represents that its use would not infringe privately owned rights. Reference herein to any specific commercial product, process, or service by trade name, trademark, manufacturer, or otherwise, does not necessarily constitute or imply its endorsement, recommendation, or favoring by the United States Government or the University of California. The views and opinions of authors expressed herein do not necessarily state or reflect those of the United States Government or the University of California, and shall not be used for advertising or product endorsement purposes.

This report has been reproduced directly from the best available copy.

Available to DOE and DOE contractors from the Office of Scientific and Technical Information P.O. Box 62, Oak Ridge, TN 37831

Prices available from (615) 576-8401, FTS 626-8401

Available to the public from the

National Technical Information Service

U.S. Department of Commerce

$$
5285 \text { Port Royal Rd., }
$$

Springfield, VA 2216 


\section{DISCLAIMER}

Portions of this document may be illegible in electronic image products. Images are produced from the best available original document. 


\title{
Modeling of MHD equilibria and current profile evolution during the ERS mode in TFTR
}

\author{
E. B. Hooper, L. D. Pearlstein and R. H. Bulmer \\ Lawrence Livermore National Laboratory \\ Livermore, CA 94551
}

July 18,1996

\section{Summary}

TFTR experiments on the enhanced reversed shear (ERS) mode ${ }^{1}$ have demonstrated particle and ion thermal diffusivities in the region of negative shear which are equal to or less than the neoclassical values. Similar enhancements have been observed in reversed central shear discharges in the shaped DIII-D geometry. ${ }^{2}$ These results, if sustained over times long compared with current diffusion times, offer the opportunity of an improved reactor. We are modeling the evolution of the TFTR ERS mode using Corsica, ${ }^{3}$ a predictive 1-1/2 D equilibrium code. Similar modeling is being done for DIII-D; ${ }_{;}^{4}$ the common goal is to better understand the physics of the discharges in order to predict performance and eventually to provide a capability of real-time control of the profiles.

Here we describe a first step in applying Corsica to the TFTR discharges. We first examine the equilibria generated in TRANSP, using the output pressure and safety factor, $q$, (or the parallel current) profiles to regenerate the magnetic equilibria. Two TRANSP options are used: (1) A minor radius-like coordinate is used as a flux surface label, or (2) Toroidal flux is used to label the surfaces. Our equilibria agree much better with option (1) than (2). However, we still find incompatibilities among the profiles, viz. fixing the $\mathrm{q}$ and $p$ profiles yields a current profile somewhat different from TRANSP.

The second step in the analysis presented here is to compare the time evolution of the $q$ and current profiles with experiment. The calculation is initialized at a time before the neutral beams are ramped up; the evolution is followed through the reverse central shear period, using as inputs the pressure and current drive results from the TRANSP analysis. The calculation is thus an evaluation of the magnetic field diffusion due to neoclassical resistivity; the result is compared with the experimental results. The calculated $q$ profiles agree reasonably well $/$ with experiment, as will be detailed below.

We wish to acknowledge the assistance of G. L. Schmidt and R. M. Wieland in carrying out the analyses described here, especially in providing access to TFTR data and understanding. 


\section{Corsica}

Corsica is a comprehensive suite of codes, integrated together under a common shell designed to handle disparate time and spatial scale physics without being constrained by the fastest time or finest spatial scales. For our present purposes we use the MHD equilibrium solver, $\mathrm{TEQ}^{5}$ and transport packages. Also available, but not used here, is an edge physics code (UEDGE) which is coupled to the toroidally confined plasma.

TEQ solves the Grad-Shafranov (GS) equation, given an input description of the pressure, $p(\psi)$, and information determining the parallel current profile, e.g. $q(\psi)$ or $\langle\mathrm{j} \bullet \mathrm{B}\rangle /\left(R_{0}\langle\mathrm{~B} \bullet \nabla \varphi\rangle\right)$, with the angular brackets indicating a flux-surface average and $R_{0}=265 \mathrm{~cm}$. The parallel current profile data are used to determine the usual toroidal field function, $F(\psi) \equiv R B_{\text {tor }}$, required in the GS equation. The code can use either fixed or free boundaries and calculates equilibria with either a direct or inverse solver.

TEQ has been benchmarked against EFIT and agrees well with JSOLVER. In addition, it has been successfully benchmarked against a suite of international codes during the ITER design activities, providing confidence in the results. ${ }^{6}$

Although the transport module includes the full set of neoclassical processes as well as a broad set of transport models, only the magnetic field diffusion resulting from neoclassical electrical conductivity is turned on during the profile evolution calculations presented here. The total plasma current is constrained to match the experimentally measured value.

Bootstrap current is included with the neutral beam current drive input from the TRANSP calculations for each discharge. Future calculations using Corsica will include calculations of the bootstrap current and the neutral beam current drive.

The TFTR geometry has been described in detail in the code, as shown in Fig. 1. Coil currents are determined from the experiment, and the outer surface fixed and constrained by the point of limiter contact. In the discharge considered here, the inner limiter was determining.

\section{TFTR data input}

Input data is in the form of "UFILES" from the TFTR database, which are read into Corsica. Machine data, such as coil currents and the total plasma current, are extracted from the TFTR waveform base. Plasma profile data is extracted from an appropriate TRANSP run for the discharge of interest. The MHD equilibrium requires the total (kinetic) pressure, $p$, and either $q$ or $\langle\mathbf{j} \bullet \mathbf{B}\rangle /\langle\mathbf{B} \bullet \nabla \varphi\rangle$. In addition, densities, temperatures, $Z_{\text {eff }}$ etc., required for the evolution calculation, are extracted from TRANSP and read into Corsica.

In the following, we consider discharge 84011, which was one of the discharges analyzed in the TFTR ERS publication. ${ }^{1}$ 


\section{MHD equilibrium calculation}

Because the equilibrium calculation uses current and pressure profiles from TRANSP, the equilibrium calculated by Corsica should be identical with the input. However, our initial calculations showed significant differences with equilbria calculated using the MHD option, VMEC. Specifically, we found that the three input profiles (safety factor, current density, and kinetic pressure) are inconsistent and that the major radii of the flux surfaces do not match those from the TRANSP runs. The disagreement is particularly bad in the vicinity of the major axis. The effect was especially strong late in the discharge when the ERS mode is highly developed. Accordingly, new TRANSP runs were generated ${ }^{7}$ for the purpose of determining the effect of the chosen flux variable. Here we consider two of the runs:

84011W03 The MHD equilibrium was calculated using the VMOMS option in TRANSP, which has an adaptive mesh which uses a minorradius like coordinate.

84011W04 The MHD equilibrium was calculated using the VMEC option in TRANSP, which uses an expansion in toroidal flux, so that near the magnetic axis the variable is essentially varying as the square of the minor radius. The calculation used in these comparisons had 101 mesh points.

To illustrate the results, we compare the equilibria at 2.70 seconds. Corsica was run to fit the $q$ and (kinetic) pressure profiles from TRANSP. Figure 2 shows results for $84011 W 04$. Note the large difference in the fluxsurface centers near the origin. The results for 84011 W03 are shown in Fig. 3. Here, the two flux-surface centers agree to within $1 \mathrm{~cm}$ throughout the plasma. Interestingly, the calculated result from W04 is in good agreement with the two results in W03.

Following a suggestion by Greg Schmidt, we examined the visible bremsstralung data for this discharge. It suggests that the actual magnetic axis may be less by several centimeters than any of these calculated here. Although this issue lies outside the present comparison, it may have consequences for understanding the stability of the plasma in the reversedshear configuration.

\section{Profile evolution}

To follow the evolution of the safety factor and current profile, we started with the equilibrium (84011W03) at $2.01 \mathrm{sec}$. Corsica matched the $q$ - and $p$ profiles, as in Figs. 2 and 3. The large surface current present in the TRANSP output, presumably due to the fitting used for the MSE data ${ }^{8}$, was smoothed by evolving the equilibrium (magnetic field diffusion) for $10 \mathrm{~ms}$ with fixed density, $Z_{\text {eff }}$ temperature, kinetic pressure, and current drive, taken equal to 
the TRANSP output at $2.01 \mathrm{sec}$. (The current drive included both neutral beam and bootstrap currents.) The evolution was then restarted using these as time dependent inputs, but with the electrical conductivity and magnetic field diffusion determined by the neoclassical conductivity through the solution of Ohm's law.

Results are shown in Figs. 4-8. Several features are immediately apparent: The general features of the experimental profiles are reproduced by the calculations. The calculated safety factor on axis, $q(0)$, is slightly higher than the data, by a factor $<10 \%$ except for a transient in the data at about 2.4 $\mathrm{sec}$. The calculated minimum in the q-profile is slightly deeper and at a slightly larger radius than the data. The peak in the q-profile which appears in the data near the magnetic axis at late times is reproduced by the calculation.

\section{Discussion and next steps in modeling ERS discharges}

Several conclusions follow from the calculations to date:

1. The MHD flux surfaces near the magnetic axis are in better agreement with TRANSP when it uses VMOMS to determine equilibria. In fact, the Corsica calculation of the flux surface centers starting with VMEC, agree better near the magnetic axis with VMOMS than with the starting equilibrium.

2. If the Corsica safety factor profile is matched with TRANSP, the current density profile differs in detail although it has the same general structure and magnitude.

3. The time evolution of the magnetic field due to neoclassical diffusion is in reasonable agreement with that observed, when the neutral beam current drive and bootstrap current are used from TRANSP.

The next step to be undertaken in the calculation is to evaluate the bootstrap current from neoclassical theory and to determine the neutral beam current drive from the Corsica model. The calculated evolution of the equilibrium using the density, temperature, and kinetic pressure (including fast particles) as input will be tested against the experimental data.

\section{Acknowledgments}

We wish to acknowledge the assistance of the Princeton Plasma Physics Laboratory staff in this work. The work was supported by the U.S. Department of Energy under Contract No. W-7405-ENG-48. 


\section{References}

1. F. M. Levinton, M. C. Zarnstorff, et al., Phys. Rev. Letters 75, 4417 (1995).

2. E. J. Strait, L. L. Lao, M. E. Mauel, B. W. Rice, T. S. Taylor, et al., Phys. Rev. Letters 75, 4421 (1995).

3. J. Crotinger, et al., Bull. Am. Phys. Soc. 38, 2016 (1993).

4. T. A. Casper, J. Crotinger, W. Meyer, J. Moller, L. D. Pearlstein, B. Rice, B. Stallard, L. Lao, and T. Taylor, 23rd Europ. Phys. Soc. Conf. Control. Fusion and Plasma Phys., Kiev, June 24-28, 1996.

5. For a description see L. L. LoDestro and L. D. Pearlstein, Phys. Plasmas 1,90 (1994).

6. L. Zahkarov (Princeton Plasma Physics Laboratory), private communication.

7. R. M. Wieland provided the TRANSP calculations.

8. S. P. Hirshman, et al., Phys. Plasmas 1, 2277 (1994). 


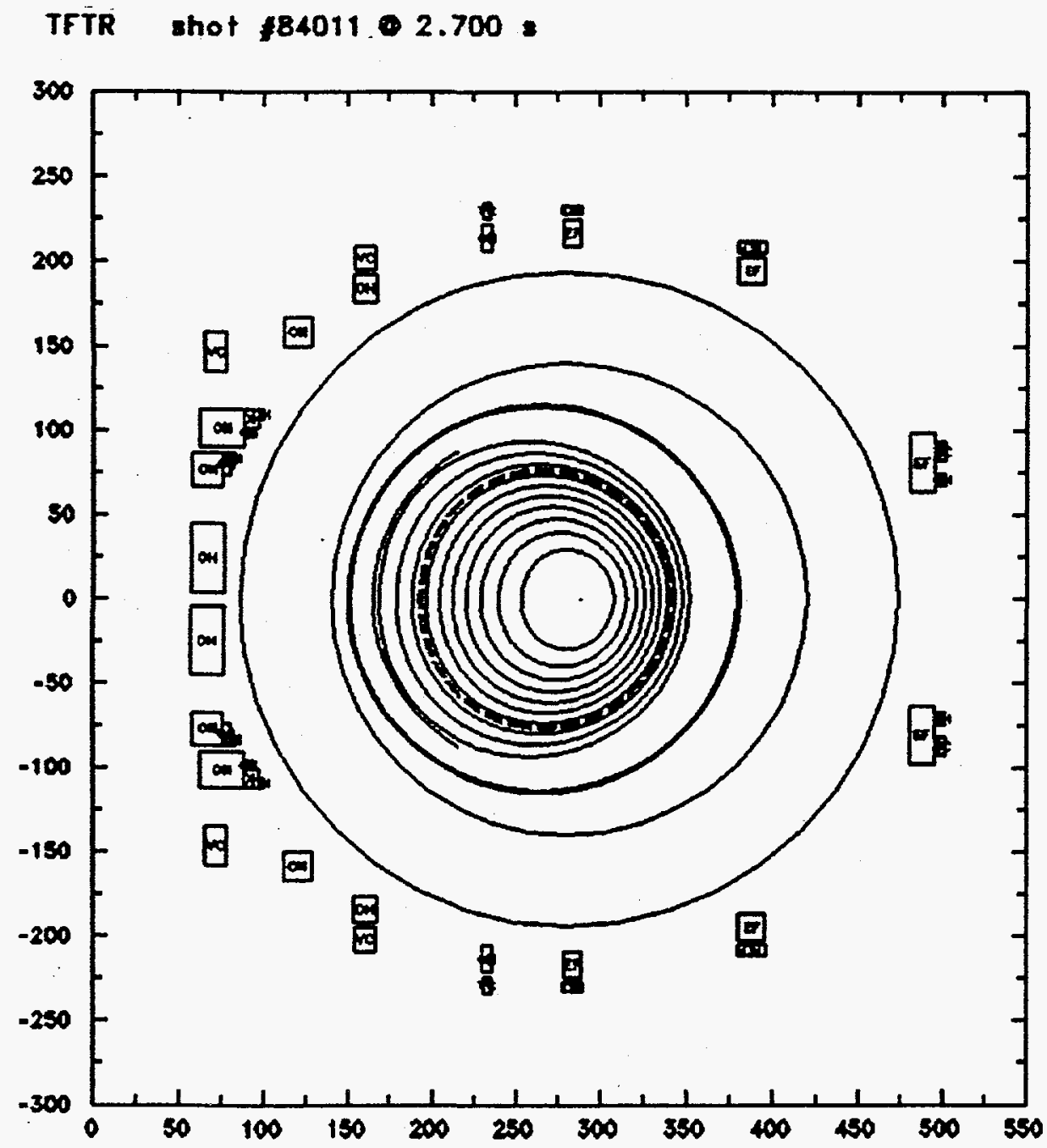

Fig. 1. Model of TFTR used in the CORSICA calculations. Shown are the poloidal and toroidal field coils, the vacuum system wall, the inner limiter, and flux surfaces corresponding to TRANSP run $84011 W 03$ at 2.7 seconds. Note that the plasma is in contact with the inner limiter at a single point. 

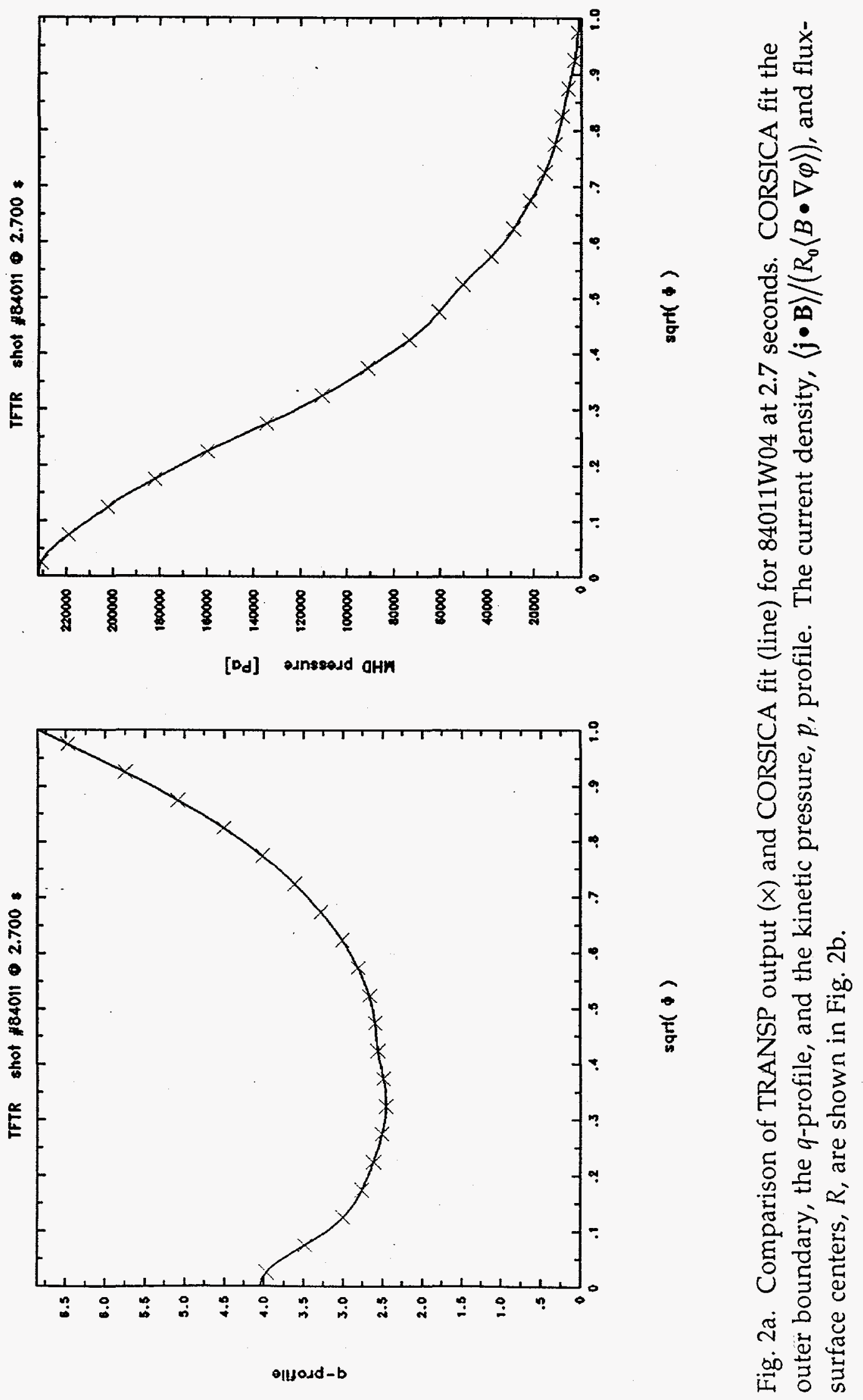


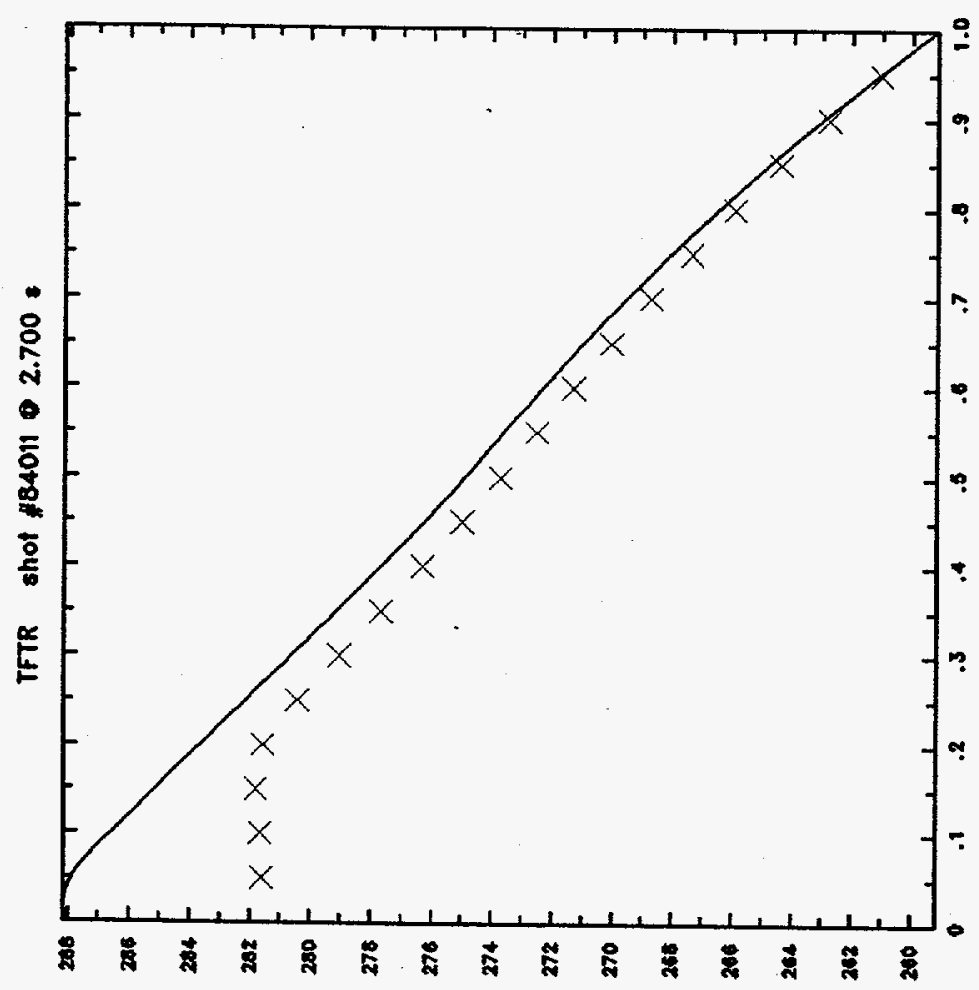

[wo] sepuso y

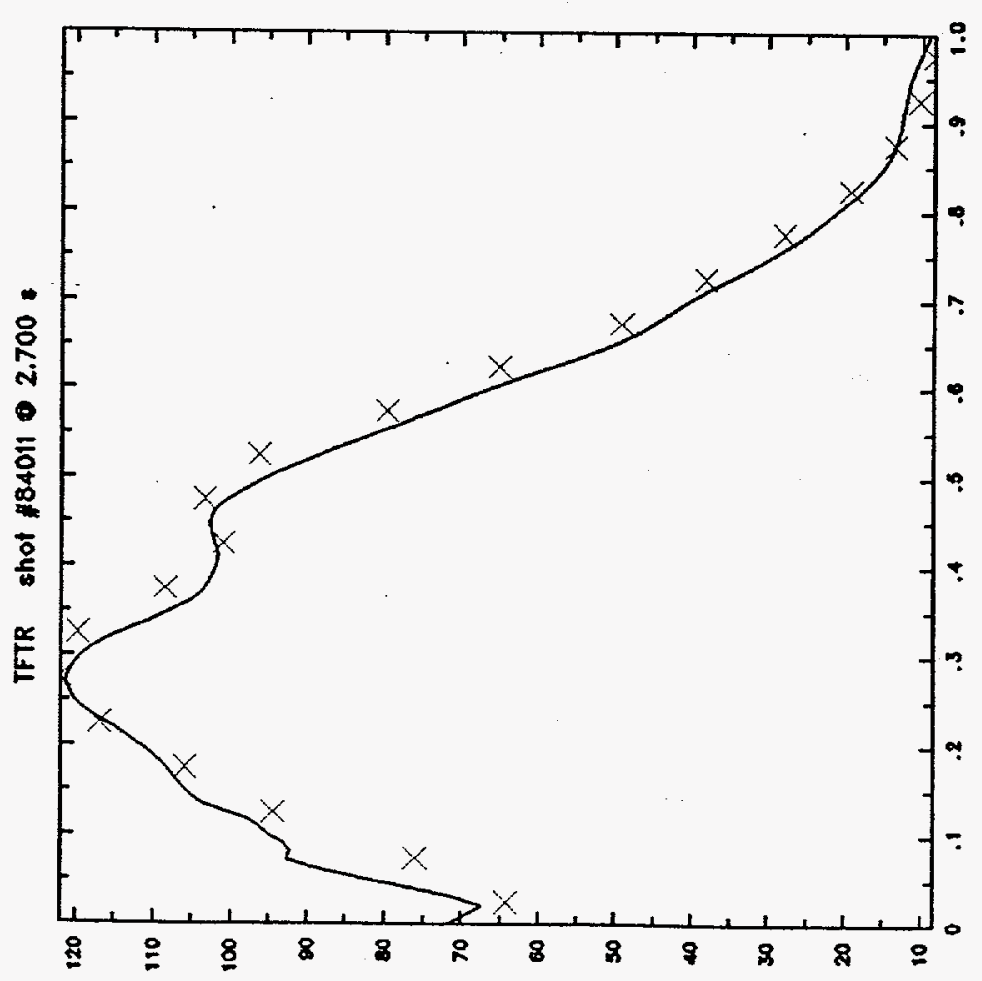

$\left[Z_{m=u_{0}} / \nabla\right]$ oy $/\langle\phi p D \beta B \cdot \theta\rangle /\left\langle g^{\prime} r\right\rangle$

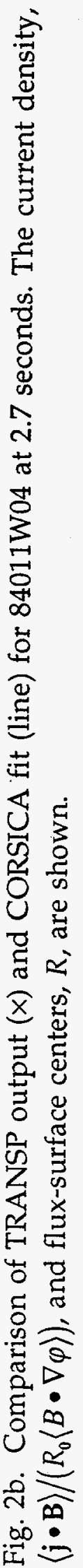



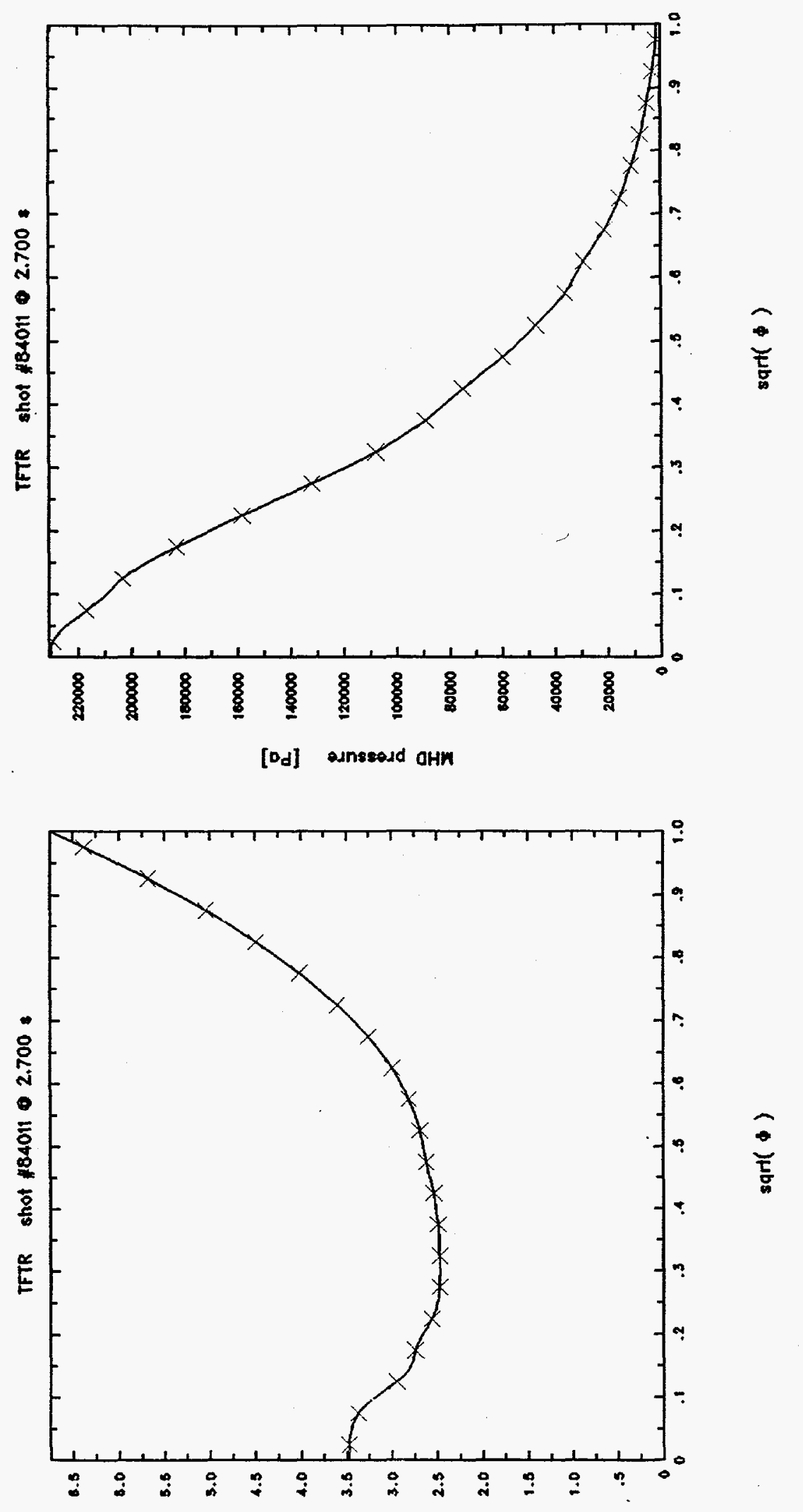

9lLodd-b

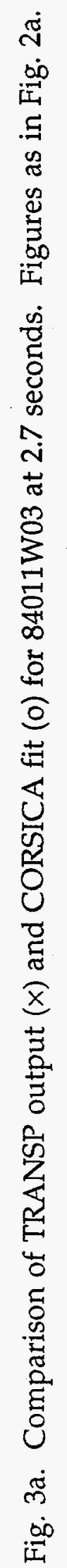



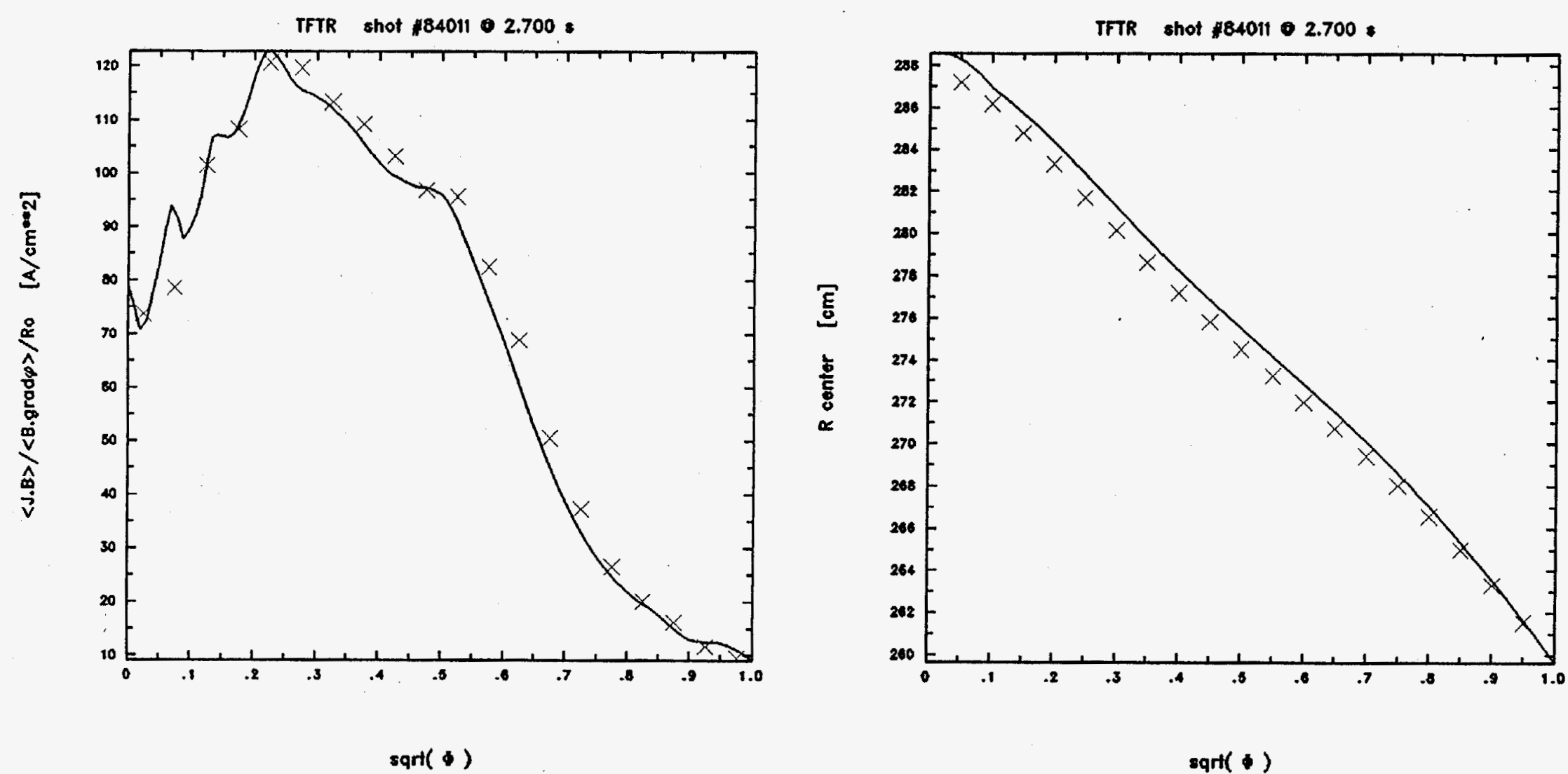

Fig. 3b. Comparison of TRANSP output $(x)$ and CORSICA fit (line) for 84011W03 at 2.7 seconds. The current density, $\langle\mathrm{j} \bullet \mathrm{B}\rangle /\left(R_{0}\langle B \bullet \nabla \varphi\rangle\right)$, and flux-surface centers, $R$, are shown. 


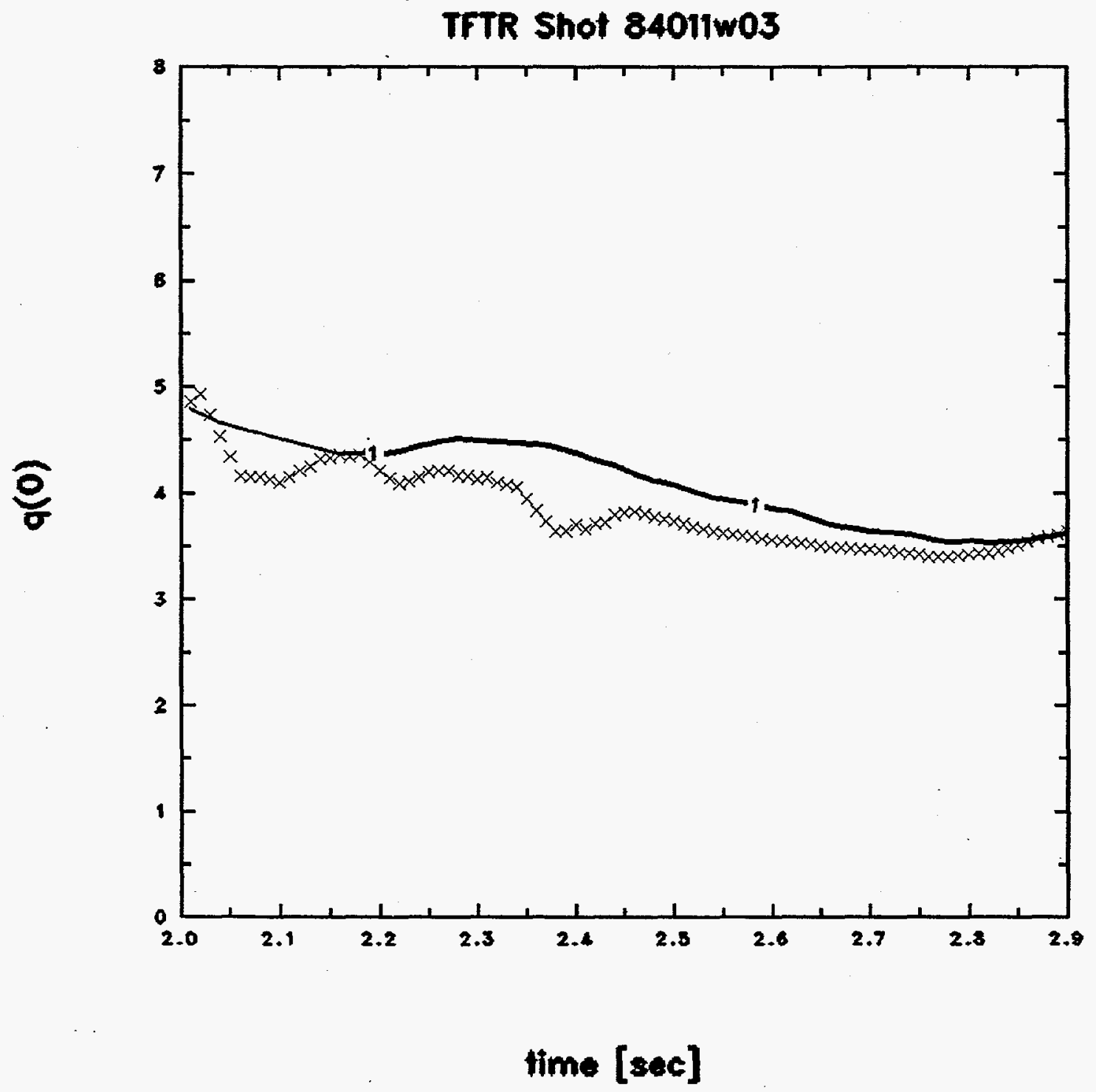

Fig. 4. Time history of the safety factor on axis. TRANSP (84011W03) is shown by crosses and CORSICA by the line. 


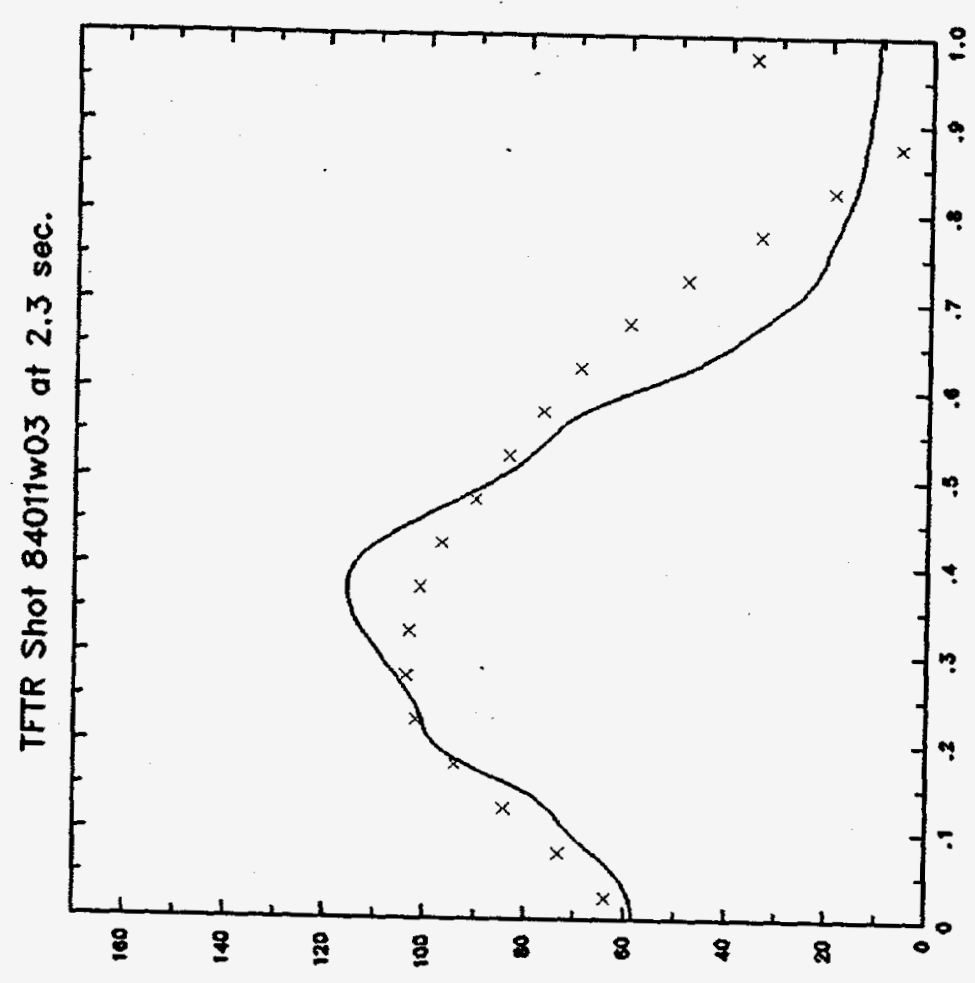

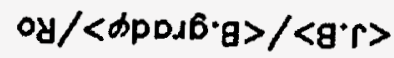

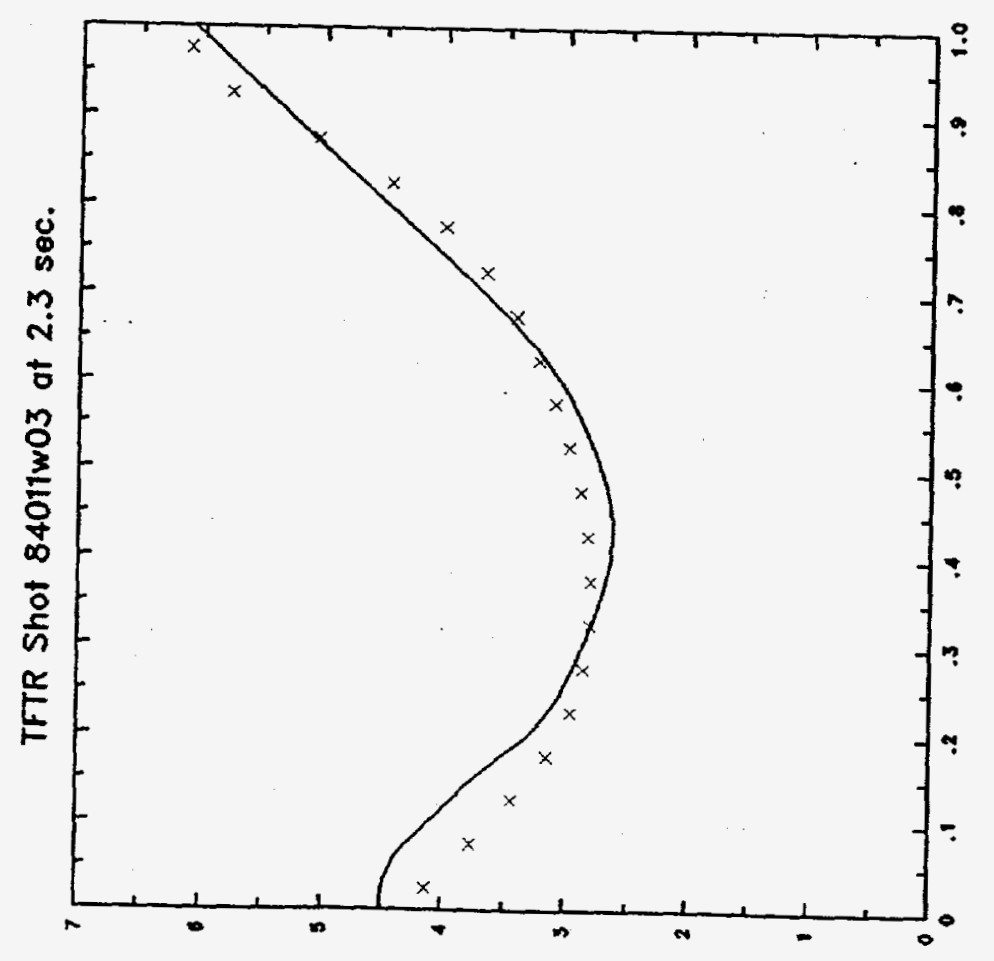

b

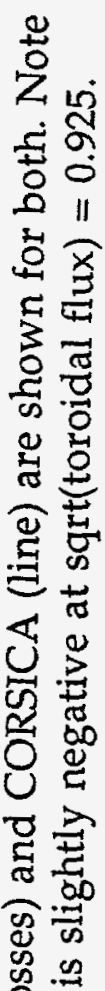

怘

氖兑

要

足

हू

.

造

के

ن

$+7$

过

这

운

Q

艺严

造

0 in

롫

ส

苍雚

党畩

in क्ष

它菜 

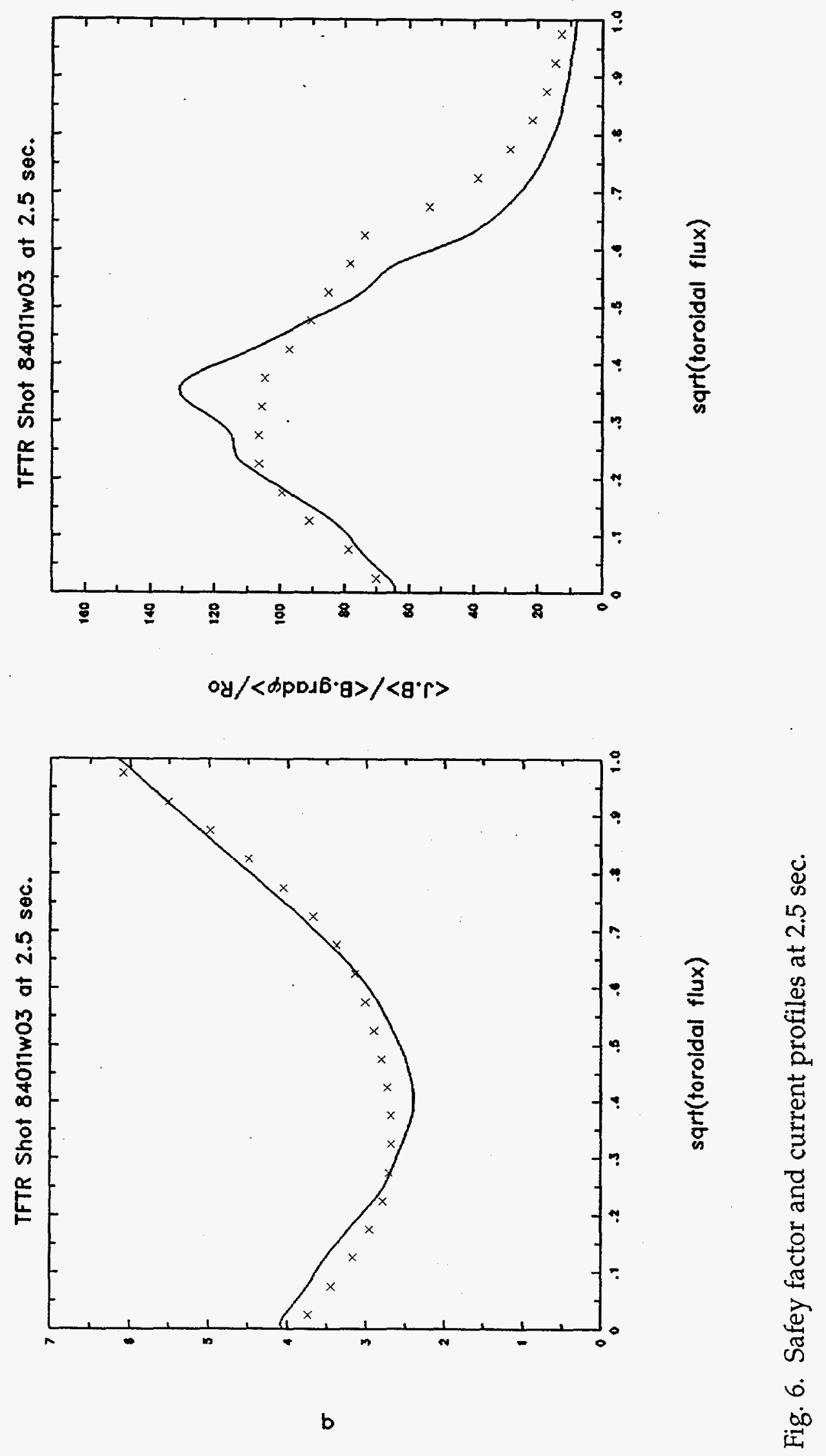

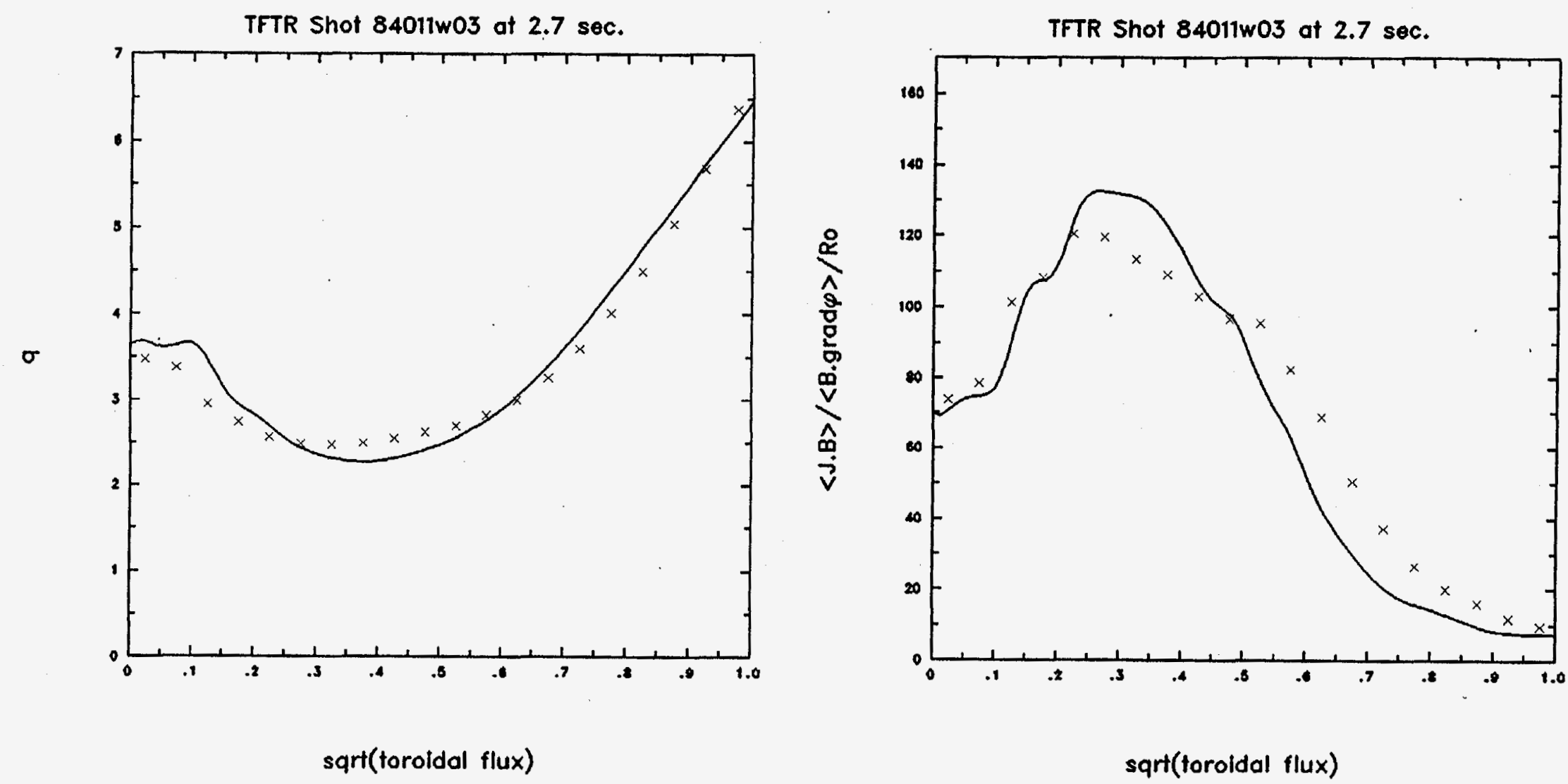

Fig. 7. Safety factor and current profiles at $2.7 \mathrm{sec}$. 


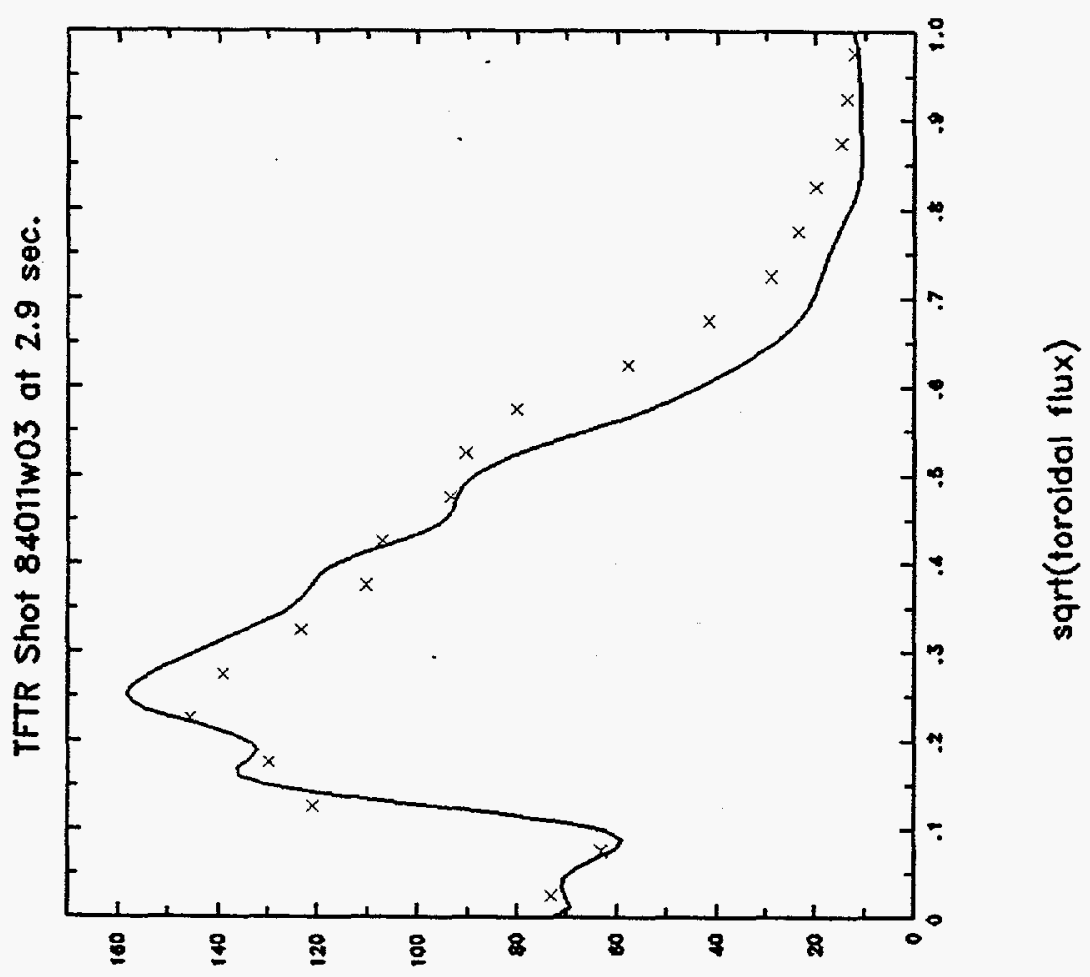

oy $/\left\langle\phi p D J 6 \cdot g>/<\theta^{\prime} r\right\rangle$

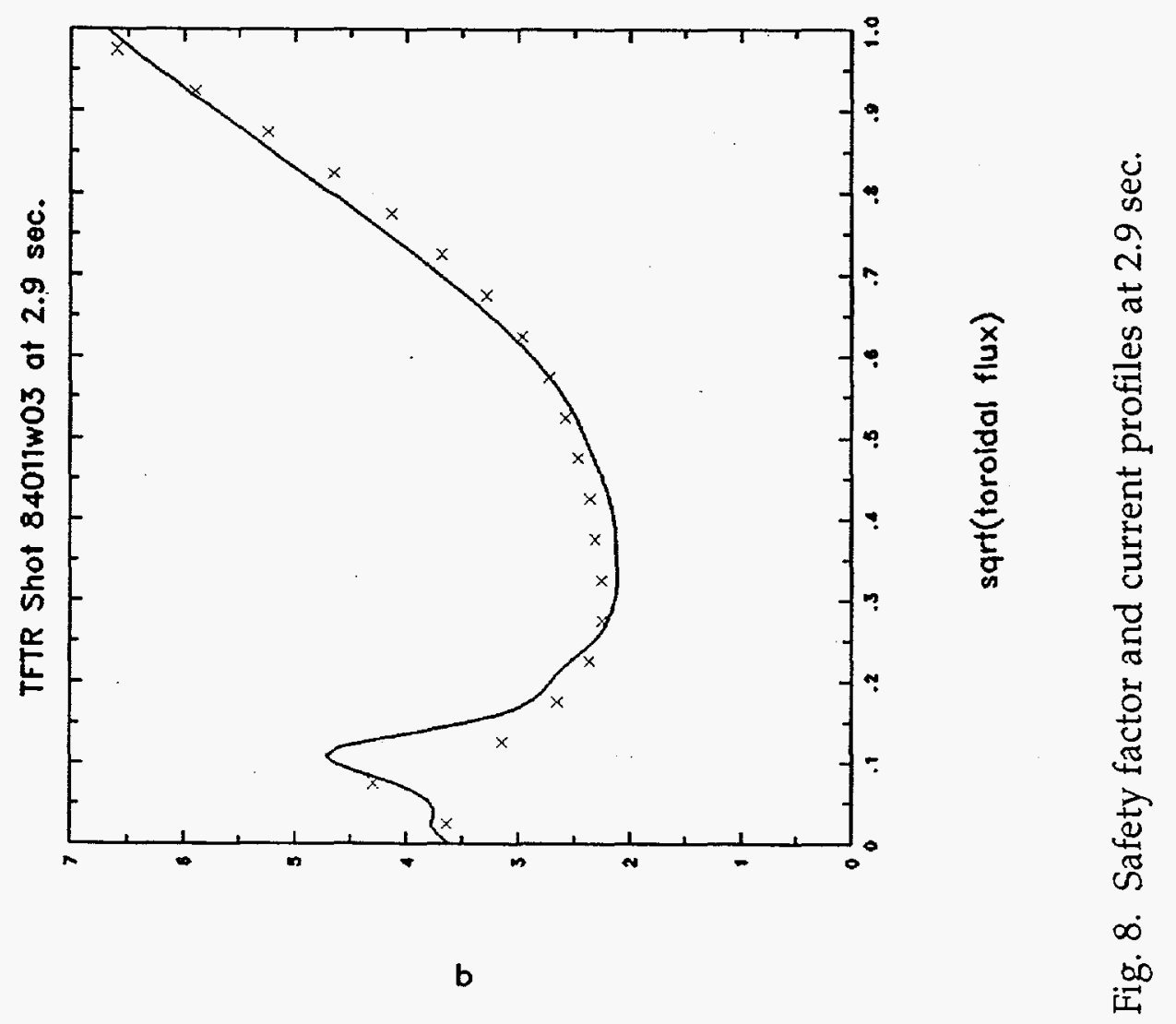

